\title{
Biodegradable Polymers and their Role in Drug Delivery Systems
}

\author{
Kiran Dhaliwal* and Priya Dosanjh \\ UCL Centre for Nanotechnology and Regenerative Medicine, Division of Surgery \& Interventional Science, United Kingdom
}

Received: 谧: November 05, 2018; Published: 無: November 16, 2018

*Corresponding author: Kiran Dhaliwal, UCL Centre for Nanotechnology and Regenerative Medicine, Division of Surgery \& Interventional Science, Royal Free London NHS Foundation Trust, Pond Street, London NW3 2QG, United Kingdom

\begin{abstract}
Biodegradable polymers are currently limited in their application in engineering of soft tissues because they are too stiff and incompliant. Biodegradable elastomers overcome these limitations; they have ability to recover from multiple deformations. Citric Acid based Biodegradable Polymers (CABEs) are a family of elastomers that have been recently developed. Further developments in this area are still needed but they provide a promising new material that can be used for drug delivery systems.
\end{abstract}

\section{Biodegradable Polymers}

Biodegradable polymers are polymers that breakdown within a limited period of time after being placed in the body and are designed to offer temporary support. With an estimated consumption of 68 million kilograms per year in 2001 [1], biodegradable polymers are widely used as biomedical devices and in tissue engineering applications. Biodegradable polymers can either be natural or synthetic. The use of synthetic polymers over naturally occurring materials provides several advantages. Firstly, synthetic polymers can be synthesised via a method that can be reproduced to give the same polymer, with the exact same composition each time. They have unlimited availability and can be produced with a wide range of physical, chemical and mechanical properties that can be modified depending on the application.

The most widely used synthetic biodegradable polymers belong to the polyester family, such as Poly Lactic Acid (PLA) and Poly Glycolic Acid (PGA) and their copolymers, such as PGLA. They have been extensively studied and reported in the literature and have many applications including resorbable sutures [2], surgical fixation devices [3] and drug delivery devices. Other commonly used biodegradable polymers include polydioxanone, which are primarily used as a suture material, marketed as Ethicon. The majority of the cell and drug delivery systems that have been developed are formed using biodegradable polymers. Controlling the release of biologically active molecules is of the uttermost importance in tissue engineering; tissue repair and regeneration is a complicated process and requires a large number of associated growth factors and hormones. Thus, delivery systems that can provide controlled release of these factors are crucial to the success of tissue engineering [4]. Their importance is also recognised in drug delivery systems where the development of novel systems has been made possible largely by the advances in polymer science.

The use of biodegradable polymers offers several advantages over other materials. Important advantages include the ability to tailor the mechanical properties, the degradation rates and the ability to be formed into various shapes. The main advantage of biodegradable polymers is that their degradability reduces the need for subsequent surgical removal, saving time and money. However, the creation of degradation products can also be problematic. Taylor et al. [5] reported that the degradation products of PLA and PGA were highly toxic if they accumulated. This is because the degradation products are acidic in nature. The result can be a high local acid concentration, which can trigger an undesirable local inflammatory response. This is more of a problem if a larger implant is used, as in many orthopaedic applications [3]. They can also be complicated and expensive to synthesise and process, especially if further modifications are needed.

The main limitation of the commonly used biodegradable polymers, such as $\alpha$-hydroxy acid and polydioxanones, is that they are too stiff and incompliant making them incapable of reversible deformation. Furthermore, they do not resemble the physical properties of human soft tissue. Their use can lead to irritation, inflammation and scar tissue formation; consequently, preventing 
integration of the device within the body. Thus, their success in tissue engineering applications is limited [6].

\section{Biodegradable Elastomers}

This lack of suitable scaffold materials for tissue engineering led to the development of more elastic biodegradable polymers biodegradable elastomers. Elastomers are polymers that exhibit resilience and the ability to stretch and retract rapidly. Due to their elastic nature they are able to sustain and recover from multiple deformations without causing irritation to surrounding tissues. The chief reason that elastomers are successful where biodegradable polymers are not stems from their elasticity; this allows them to recover from multiple deformations and makes them more similar to organic human tissue. Furthermore, the mechanical properties of elastomers can be matched to specific soft tissue structures within the body and degradation rates can be tailored to maximise tissue incorporation.

This improves tissue regeneration since it allows the gradual transfer of stresses from the degrading scaffold to the newly formed tissue. Biodegradable elastomers have been described as a "valuable strategy for satisfying the requirements of regenerative medicine and tissue engineering" [7]. A recently developed biodegradable elastomer that has been successful in tissue engineering applications is Poly Glycerol Sebacate (PGS). In 2002 Wang et al. [6] designed, synthesised and characterised the novel biodegradable elastomer PGS. In comparison to biodegradable polymers, PGS demonstrates better biocompatibility; it produces significantly less inflammation and fibrosis. This study is frequently cited which highlights the impact of this work. PGS has since been studied extensively and has proven to be successful in many applications, such as drug delivery [8], supporting the growth of a variety of cells and even wound healing [9].

\section{Photo-Polymerisation}

To realise the full capacity of a material it must be easily processed. It is especially important to be able to use mild techniques that will not damage molecules, particularly where the inclusion of active molecules within the polymer are envisaged. One alternative processing strategy that has been investigated is photo-polymerisation. Photo-polymerisation is the use of light to initiate crosslinking of a molecule. The main advantage of photopolymerisation is that it allows biologically active molecules to be incorporated within the polymer without being exposed to harsh processing conditions because it can be carried out at physiological temperatures. Harsh processing conditions such as high temperatures or cytotoxic organic solvents limits the in situ application of polymers, as well as their use as cell or drug delivery systems. This highlights the large, unmet need for milder, gentler processing methods; a problem which can be overcome by using photo-polymerisation. Furthermore, photo-polymerisation can occur directly in or on the tissue, providing the advantage of localised delivery, ease of application and reduction in dosage amount if used as a delivery vehicle.

Hubbell et al. were the first to demonstrate in vivo photopolymerisation of degradable polymers. They successfully demonstrated that it could be used for the prevention of postoperative adhesions. Further examples include: Hubbell et al. [10] who investigated PEG-oligoglycolyl-acrylates as a controlled release carrier and Nivasu et al. [11] who looked at controlled release of the antibiotic sulfamethoxazole.

\section{Citric Acid Based Biodegradable Polymers (CABEs)}

Citric acid based biodegradable polymers CABEs are a family of polyester elastomers that have been recently developed. Poly(diol citrates) were the first group of CABEs to be synthesised and have been most widely studied. Synthesis of poly(diol citrates) is based on polymerisation of a linear diol with citric acid (Figure 1). Citric acid (Figure 2) is a multifunctional molecule which provides pendant functionality and is a non-toxic by-product of the Krebs cycle. It is FDA approved and currently used in clinical settings as an anticoagulant [12]. The primary advantage of CABEs over other polymers is their ease of synthesis. They are formed via poly-condensation reactions without the use of toxic catalysts or crosslinking reagents [13]. The monomers are also readily available, relatively inexpensive and importantly non-toxic, even once they have been degraded. They are also synthesised in very mild conditions in a short space of time. CABEs can be synthesised at temperatures as low as $135 \mathrm{oC}$ in a reaction taking only 125 minutes, whereas many of the biodegradable polymers reported in the literature have very complex and costly synthesis procedures. For example, the synthesis of Polyhydroxyalkanoates (PHAs) [14] has a very complicated fermentation synthesis procedure and the reactions to form polyesters such as PGLA can take up to six hours and requires temperatures of up to 200oC. Furthermore, the formation of elastomers from polymers of D,L-lactide [15] and the synthesis reaction for PGS can both take up to three days to complete [13].

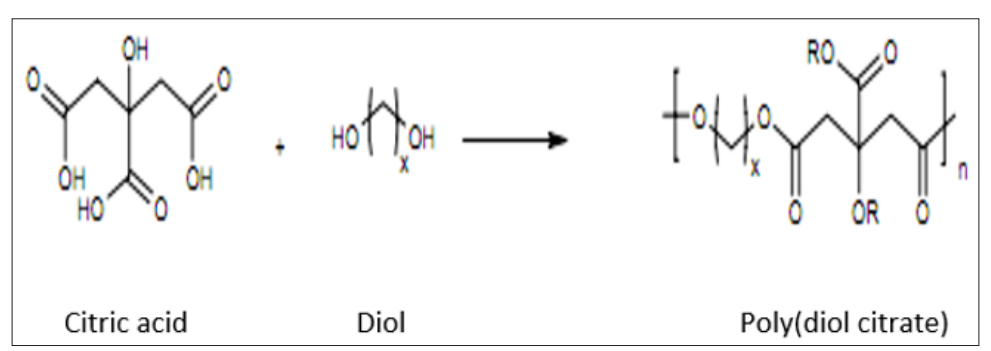

Figure 1: Reaction for Poly(diol citrate) synthesis. 


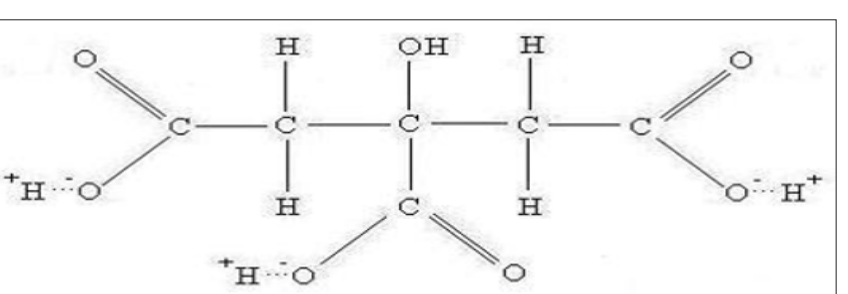

Figure 2: Chemical structure of citric acid monomer.

The poly-condensation reaction produces a crosslinked polyester network with degradable ester bonds [16] and the elastic nature of the molecule is conferred by this crosslinked network. The reaction preserves the pendant functionality of the citric acid molecule for potential conjugation with biological molecules without the need for further modification. This is because citric acid is a multifunctional molecule; it removes the need for further modification of the polymer, saving time and money. The low cost of the monomers and the simple synthesis procedure of CABEs increases their potential for commercialisation. This is an important consideration that can affect the clinical implementation of materials. One of the most unique aspects of CABEs is ease with which their physical properties can be controlled. These properties have been shown to be controllable by

i) Changing the diol used [13]

ii) Changing the molar ratio of the monomers [17],

iii) Changing the synthesis conditions, including temperature [17] and

iv) Changing the polymerisation conditions [7]. Modification of these conditions can also modulate degradation rates [13]; this allows CABEs to be tailored to specific applications unlike other biodegradable polymers.

\section{Development of CABEs}

The first CABE was synthesised by Yang et al. [13]. Citric acid was reacted with a range of diols, including 1,8-Octanediol, to create the novel Poly(1,8-Octanediol- Co-Citric Acid) (POC) biodegradable elastomer. POC demonstrates good mechanical properties with an ultimate tensile strength as high as $6.1 \mathrm{MPa}$ and Youngs modulus with a range of 0.92-16.4 $\mathrm{MPa}$, making it within the range of many soft tissues in the body. Yang also demonstrated that POC had excellent biocompatibility; it supports the attachment and proliferation of Human Aortic Smooth Cells (HASMC) and Human Aortic Endothelial Cells (HAEC). The cells were able to attach to the surface without needing any pre-treatment or surface modification. POC also demonstrated an in vivo inflammatory response similar to PLGA, a degradable polymer widely accepted as biocompatible [14-17].

Since their initial development, they have been extensively studied and further research has led to a number of new key developments Owing to their unique properties CABEs have been utilised in a wide variety of applications; the most successful being cardiovascular tissue engineering. Yang et al. developed a biphasic tubular POC scaffold for use as small diameter blood vessel grafts. The mechanical properties of the scaffold are very similar to properties of native human arteries and veins. In addition, Kibbe et al. [18] provides evidence for POC as a non-thrombogenic coating for blood contacting devices. Kibbe et al. demonstrated that when current ePTFE grafts are coated in POC it resulted in improved endothelial cell adhesion and reduced platelet adhesion compared to standard ePTFE grafts. Since their initial development their repertoire has greatly expanded due to further research in the area owing to the promising results initial studies into CABEs have produced.

\section{Drug Delivery Systems (DDS)}

Drug delivery is a fast growing field with an unmet need for more effective materials for drug delivery. Drug delivery systems overcome the limitations imposed by conventional delivery routes such as oral, intravenous and intramuscular, which prevent effective or efficient drug delivery. Conventional delivery routes typically release a large amount of the drug initially, over a very short period of time (Figure 3). There is then a decline in the plasma concentration of the drug and administration of another dose becomes necessary to return the plasma concentration of the drug to therapeutic levels. This results in multiple administrations of the drug since the therapeutic dose cannot be sustained (Figure 4).

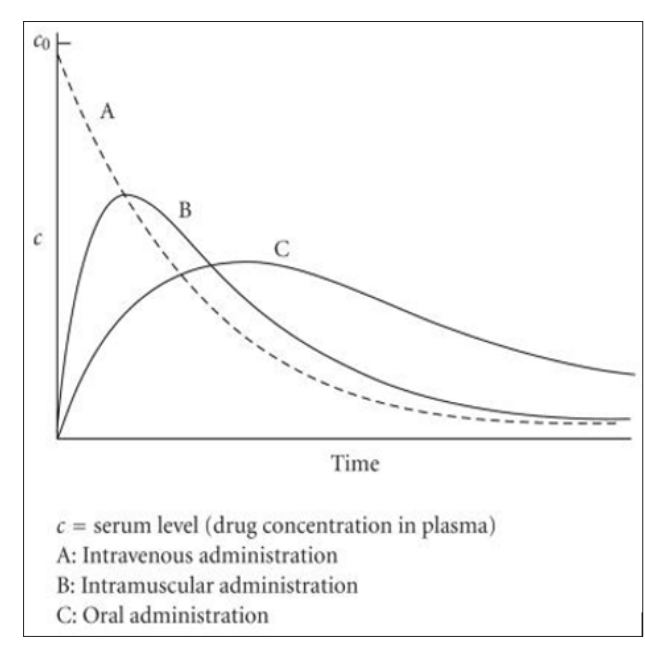

Figure 3: Conventional drug release profiles for intravenous, intramuscular and oral administration.

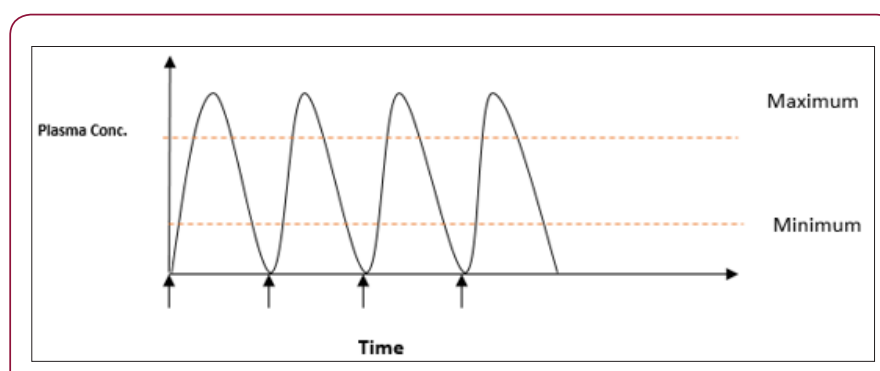

Figure 4: Conventional drug release profiles for intravenous, intramuscular and oral administration showing repeated dose administration (indicated by arrows) over time. 
This can be difficult for patients to maintain and has several disadvantages. Firstly, patients need to have frequent repeat doses of the drug which can be inconvenient and time consuming, resulting in reduced patient compliance. Furthermore, a large dose of the drug has to be administered because by the time it reaches the site of action only a small percentage of the initially administered dose remains. Consequently, a high concentration of the drug remains in the systemic circulation and, depending on the drug, this can cause a variety of unwanted side effects. This is particularly important if the drug is highly toxic, such as chemo-therapeutic drugs. These conventional delivery methods are seen as inadequate for the delivery of many therapeutic agents, especially those that have very short half-lives, such as peptides, genes and recombinant proteins, and would therefore need to be administered very frequently to maintain a therapeutic dose.

Drug delivery systems provide a solution for more effective drug delivery. Gregoradis first proposed the use of carriers to deliver and release drugs in a controlled manner at a specific site in the 1970's. A primary advantage of drug delivery systems is that they allow controlled and sustained release of the drug (Figure 5). This essentially eliminates the need for patients to take multiple doses of a drug and can therefore improve patient compliance and convenience, particularly for drugs that require frequent administration. The drug can also be delivered locally, close to its site of action. This reduces the amount of drug that is needed and minimises the systemic concentration of the drug, consequently reducing the likelihood of side effects.

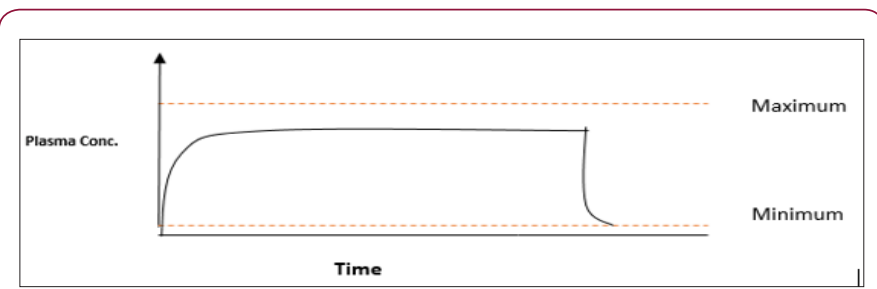

Figure 5: Typical drug release profile from a drug delivery system. There is a sustained release of the drug over time.

Furthermore, drugs that may be unsuitable for clinical use by conventional routes of administration owing to problems of stability or narrow therapeutic index can be successful used if delivered by controlled release.

\section{Polymeric Drug Delivery Systems}

Polymer science has been the backbone of the development of new drug delivery systems for the past few decades. Polymeric materials are widely used in drug delivery because they have been extensively studied and shown to be biocompatible, non-toxic and have tunable mechanical properties and degradation rates. The polymeric system can also provide a form of protection for the drug, preventing exposure to the physiological environment. Therefore, improving the stability of the drug and increasing its bio-availability. Hence the choice of polymer is one of the most important considerations.

One example of a successful polymeric drug delivery system is Norplant, an implantable contraceptive device. It was one of the first drug delivery systems to be widely used in clinical practice. However, it is a non-degradable system and therefore requires surgical removal once the drug has depleted. Its clinical use has declined, and it has been discontinued in the UK and US. This is largely attributed to the problems of removal, including the possibility of complications during the retrieval process, the risk of infection and lack of patient compliance [19]. The use of biodegradable polymers presents a far more attractive option as there is no need for surgical removal once the drug has depleted.

Many biodegradable polymers have been investigated for use in drug delivery and have established a role in controlled drug release. Aliphatic polyesters such as poly(lactic acid), poly(glycolic acid), poly(lactide-co-glycolide), poly(decalactone) and poly( $\varepsilon$ caprolactone) have been the subject of the most extensive investigations [20]. Many biodegradable polymers have been successfully fabricated into a number of devices for drug delivery including microspheres, microcapsules and nanoparticles [21,22]. There are several ways that polymers can be utilised in drug delivery, including diffusion controlled systems, swelling controlled devices and particulate systems such as polymer-drug conjugates. This study will look at chemically controlled delivery systems, where the drug will be dispersed in a biodegradable polymeric matrix and released upon degradation of the cross-linked network (Figure 6).

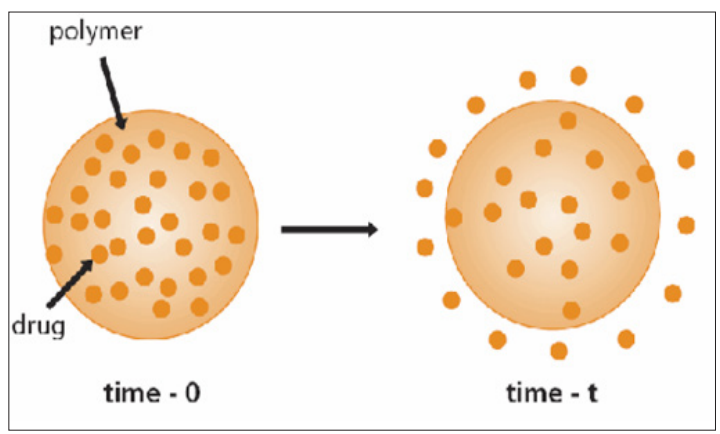

Figure 6: Schematic representation of a biodegradable drug delivery device, illustrating how the drug is released over time as the polymer degrades.

Although many new delivery methods have been developed, very few actually go on to be implemented in clinical practice. This is partly due to the problems with production. If a system is to be implemented on a large scale it must be easily produced; this is where CABEs could be successful over other delivery methods. One important problem with other biodegradable drug delivery systems it that that they often require several processing steps after drug entrapment before they can be used. In contrast, CABEs require very little processing after drug entrapment and can trap drugs under physiological conditions. This step can be a cause of drug deactivation in other systems.

There is currently only one study that has reported the use of CABEs in drug delivery. Hoshi et al. [23] used nanoporous Poly(1,8Octanediol-Co-Citrate) (POC) to form a drug delivery system to entrap Dextran, an anti-platelet drug. The results showed that POC 
could release Dextran at a slow, controlled rate and therefore could potentially be used in drug delivery applications. In addition to this, previous studies evaluating the properties of CABEs $[13,24]$ have reported that they have the potential for successful application in drug delivery but have not carried out any controlled release studies.

\section{In Situ Drug Delivery Systems}

Although in situ polymerisation has its advantages, the conditions required are very specific. The physiologically acceptable temperatures are within a very narrow range and the system must be rapidly polymerised if it is to be successfully implanted in a clinical setting [25]. The advantage of photo-initiated crosslinking is that the reaction proceeds rapidly at low temperatures and therefore may be suitable for the incorporation of thermally sensitive drugs such as peptides and proteins. Photopolymerisable systems have an advantage over other types of in situ systems, such as chemically initiated systems. This was demonstrated by Dunn et al. [26] who crosslinked biodegradable co-polymers D,L lactide and L-lactide with $\varepsilon$-caprolactone, using a chemically initiated thermo-set system, for use as a slow-release drug delivery system. Disadvantages of the system include taking up to 30 minutes to set and the highly exothermic nature of the crosslinking reaction could result in tissue necrosis. There was also burst release of the drug in the first hour which could result in the appearance of side effects [27-40].

Drug delivery systems that can be formed in situ have evolved from the need for prolonged and better control of drug administration. They offer a wide range of advantages over other drug delivery systems. Firstly, the drugs can be administered very easily by injecting into the desired site, making it quick and relatively painless for the patient, which can increase patient compliance. They also allow localised and sustained drug release over a prolonged period of time. Furthermore, it can be moulded into any desired shape, regardless of complexity, which is very difficult to achieve with other methods of drug delivery [20]. For example, PGLA microspheres are very difficult to administer because they require a surgical incision to gain access to the desired site. They also require prior preparation before they can be injected into the body, making it inconvenient for the patient [21]. In situ systems are relatively easy to synthesise, making them excellent candidates for clinical use.

\section{Conclusion}

Although many advances in polymers and drug delivery systems and methods have been made over the years, there is still a need for new, unique materials owing to the advances in pharmaceuticals. There is also a need for more gentile and facile methods of biological molecule inclusion. Photo-polymerisation provides an effective solution. Further research into this area could lead to the development of the optimal drug delivery system.

\section{References}

1. Webb AR, Yang J, Ameer GA (2008) A new strategy to characterize the extent of reaction of thermoset elastomers. Poly Chem 46(4): 13181328.
2. Middleton JC, Tipton AJ (2000) Synthetic biodegradable polymers as orthopedic devices. Biomaterials 21(23): 2335-2346.

3. Gunatillake P, Mayadunne R, Adhikari R (2006) Recent developments in biodegradable synthetic polymers. Biotechnol Annu Rev 12: 301-347.

4. Bae SE, Choi DH, Han DK, Park K (2010) Effect of temporally controlled release of dexamethasone on in vivo chondrogenic differentiation of mesenchymal stromal cells. J Controlled Release 143(1): 23-30.

5. Taylor MS, Daniels AU, Andriano KP, Heller J (1994) Six bioabsorbale polymers: In-vitro acute toxicity of accumulated degradation products. J Appl Biomater 5(2): 151-157.

6. Wang Y, Ameer A, Sheppard B, Langer R (2002) A tough biodegradable elastomer. Nat Biotechnol 20(6) 587-591.

7. Serrano MC, Chung EJ, Ameer GA (2010) Advances in applications of biodegradable elastomers in regenerative medicine. Advanced functional materials 20(2): 192-208.

8. Sun ZJ, Chen C, Sun MZ, Ai CH, Lu XL, et al. (2009) The application of poly (glycerol-sebacate) as biodegradable drug carrier. Biomaterials 30(28): 5209-5214.

9. Mahdavi A, Ferreira L, Sundback C, Nichol JW, Chan EP, et al. (2008) A biodegradable and biocompatible gecko-inspired tissue adhesive. Proceedings of the National Academy of Sciences 105(7): 2307-2312.

10. Hubbell JA, Pathak PC, Sawhney AS, Desai NP, Hill JL (1995) Photopolymerizable biodegradable hydrogels as tissue contacting materials and controlled release carriers. US5986043A.

11. Nivasu VM, Reddy TT, Tammishetti S (2004) In situ polymerizable polyethyleneglycol containing polyesterpolyol acrylates for tissue sealant applications. Biomaterials (25)16: 3283-3291.

12. Rui Shi, Dafu Chen, Quanyong Liu, Yan Wu, Xiaochuan Xu, et al. (2009) Recent advances in synthetic bioelastomers. Int J Mol Sci 10(10): 42224256.

13. Yang J, Webb A, Hageman G, Ameer G (2004) Novel citric acid-based biodegradable elastomers for tissue engineering. Adv Mater 16(6): 511516.

14. Sodian R, Sperling JS, Martin DP, Egozy A, Stock U, et al. (2000) Fabrication of a trileaflet heart valve scaffold from a polyhydroxyalkanoate biopolyester for use in tissue engineering. Tissue Eng 6(2): 183-188.

15. Gunatillake PA, Adhikari R (2003) Biodegradable synthetic polymers for tissue engineering. Eur Cell Mater 5: 1-16.

16. Tran R, Zhang Y, Gyawali D, Yang J (2009) Recent development on citric acid derived biodegradable elastomers. Recent Pat Biomed Eng 2: 216227.

17. Gyawali D, Nair P, Zhang Y, Tran RT, Zhang C, et al. (2010) Citric acidderived in situ cross-linkable biodegradable polymers for cell delivery. Biomaterials 31(34): 9092-9105.

18. Kibbe M, Martinez J, Popowich D, Kapadia M, Ahanchi S, et al. (2010) Citric acid-based elastomers provide a biocompatible interface for vascular grafts. J Biomed Mater Res A 93(1): 314-324.

19. Motlagh D, Allen J, Hoshi R, Yang J, Lui K, et al. (2007) Hemocompatibility evaluation of poly(diol citrate) in vitro for vascular tissue engineering. J Biomed Mater Res A 82(7): 907-916.

20. Madan M, Bajaj A, Lewis S, Udupa N (2009) In situ forming polymeric drug delivery systems. Indian J Pharm Sci 71(3): 242-251.

21. Jain RA (2000) The manufacturing techniques of various drug loaded biodegradable poly(lactide-co-glycolide)(PGLA) devices. Biomaterials 21(23): 2475-2490.

22. Hans ML, Lowman AM (2002) Biodegradable nanoparticles for drug delivery and targeting. Curr Opinion Solid State Mat Sci 6(4): 319-327.

23. Hoshi RA, Behl S, Ameer GA (2009) Nanoporous biodegradable elastomers. Adv Mat 21(2): 188-192. 
24. Gyawali D, Tran R, Guleserian K, Tang L, Yang J (2010) Citric acid derived photo-cross-linked biodegradable elastomers. J Biomater Sci Polym Ed 21(13): 1761-1782.

25. Hatefi A, Amsden B (2002) Biodegradable injectable in situ forming drug delivery systems. J Control Release 80(1-3): 9-28.

26. Dunn RL, English JP, Cowsar DR, Vanderbelt DP (2004) Biodegradable insitu forming implants and methods of producing the same. US5990194A.

27. Athanasiou KA, Agrawal CM, Barber FA, Burkhart SS (1998) Orthpaedic applications for PLA-PGA biodegradable polymers. Arthroscopy 14(7): 726-737.

28. Ashammakhi N, Rokkanen P (1997) Absorbable polyglycolide devices in trauma and bone surgery. Biomaterials 18(1): 3-9.

29. Bettinger CJ (2011) Biodegradable elastomers for tissue engineering and cell-biomaterial interactions. Macromol Biosci 11(4): 467-482.

30. Bryant SJ, Anseth KS (2001) The effects of scaffold thickness on tissue engineered cartilage in photocrosslinked poly(ethylene oxide) hydrogels. Biomaterials 22(6): 619-626.

31. Chitakara D, Shikanov A, Kumar N, Domb A (2006) Biodegradable injectable in situ depot forming drug delivery systems. Macromol BioSci 6(12): 997-990.

32. Dey J, Xu H, Shen J, Thevenot P, Gondi S, et al. (2008) Development of biodegradable cross-linked urethane-doped polyester elastomers. Biomaterials 29(35): 4637-4649.

\section{ISSN: 2574-1241}

DOI: 10.26717/BJSTR.2018.11.002056

Kiran Dhaliwal. Biomed J Sci \& Tech Res

(C) (i) This work is licensed under Creative

Cy Commons Attribution 4.0 License

Submission Link: https://biomedres.us/submit-manuscript.php
33. Djordjevic I, Choudhury N, Dutta N, Kumar S (2009) Synthesis and characterization of novel citric acid based polyester elastomers. Polymer 50(7): 1682-1691.

34. Gerecht S, Townsend S, Pressler H, Zhu H, Nijst C, et al. (2007) A porous photocurable elastomer for cell encapsulation and culture. Biomaterials 28(32): 4826-4835.

35. Jansen J, Boerakker MJ, Heuts J, Feijen J, Grijpma DW (2010) Rapid photo-crosslinking of fumaric acid monoethyl ester-functionalized poly(trimethylene carbonate) oligomers for drug delivery applications. J Controlled Release 147(1): 54-61.

36. Nijst CL, Bruggeman JP, Karp JM, Ferreira L, Zumbuehl A, et al. (2007) Synthesis and characterization of photo-curable elastomers from poly(glycerol-co-sebacate). Biomacromolecules 8(10): 3067-3073.

37. Qing Y, Wenying X, Rånby B (2004) Photoinitiated crosslinking of low density polyethylene I: Reaction and kinetics. Poly Engineer Sci 31(22): 1561-1566.

38. Qiu H, Yang J, Kodali P, Koh J, Ameer GA (2006) A citric acid-based hydroxyapatite composite for orthopedic implants. Biomaterials 27(34): 5845-5854.

39. Tanihara M, Suzuki Y, NishimuraY, Suzuki K, Kakimaru Y, wt al. (1999) A novel microbial infection-responsive drug release system. J Pharm Sci 88(5): 510-514.

40. Tran R, Thevenot T, Gyawali D, Chiao J, Tang L, et al. (2010) Development of a novel biodegradable elastomer featuring a dual crosslinking mechanism for soft tissue engineering. Soft Matter 6: 2449-2461.

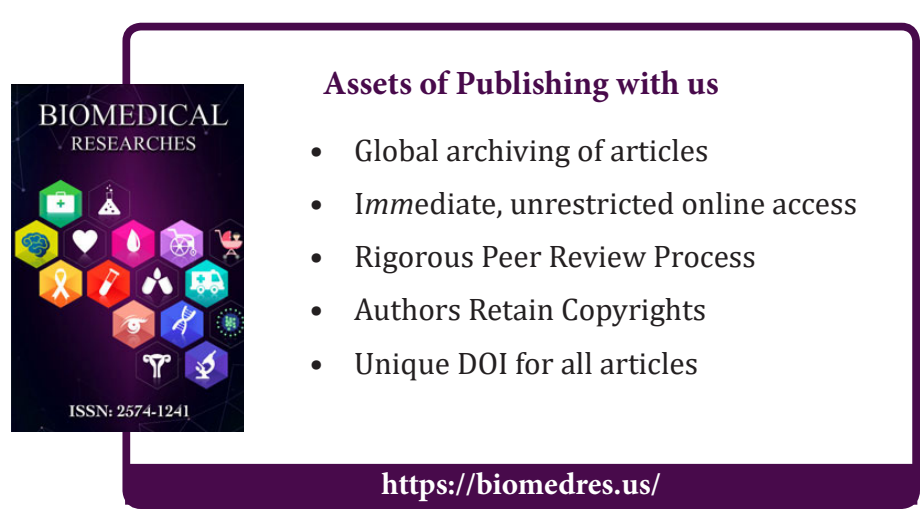

